\title{
Association of genotypes of the CYP3A cluster with midazolam disposition in vivo
}

J Miao, Y Jin, RL Marunde, C) Gorski, S Kim, S Quinney, $M$ Radovich, L Li and SD Hall
The Pharmacogenomics Journal (2012) 12, 184; doi:10.1038/tpj.2010.63

Correction to: The Pharmacogenomics Journal (2009) 9, 319-326; doi:10.1038/ tpj.2009.21; published online 9 June 2009

The list of co-authors for this article should have appeared as Miao J, Jin Y, Marunde RL, Gorski CJ, Kim S, Quinney S, Radovich M, Li L and Hall SD. At this time, the corresponding author acknowledges Dr Christopher J Gorski for his significant contribution to the research, as he participated in all of the midazolam studies in our published meta-analysis as either a principal investigator or a co-investigator.

\section{ADRA1A gene is associated with $\mathrm{BMI}$ in chronic schizophrenia patients exposed to antipsychotics}

Y-R Liu, E-W Loh, T-H Lan, S-F Chen, Y-H Yu, Y-H Chang, C-J Huang, T-M Hu, K-M Lin, Y-T Yao and H-J Chiu
The Pharmacogenomics Journal (2012) 12, 184; doi:10.1038/tpj.2011.39

Correction to: The Pharmacogenomics Journal (2010) 10: 30-39; doi: 10.1038/ tpj.2009.55; published online 17 November 2009

The above article was published with incomplete affiliations. The authors apologize for this error. The authors, with the correct affiliations, are listed below.

Y-R Liu ${ }^{1,2,11}$, E-W Loh ${ }^{1,11}$, T-H Lan ${ }^{1,3,4}$, S-F Chen ${ }^{5}$, Y-H Yu ${ }^{6}$, Y-H Chang ${ }^{1,7}$, C-J Huang, ${ }^{8}$ T-M Hu${ }^{9}, \mathrm{~K}_{-M}$ Lin $^{1}$, Y-T Yao ${ }^{2}$ and H-J Chiu ${ }^{3,10}$

${ }^{1}$ Division of Mental Health and Addiction Medicine, Institute of Population Health Sciences, National Health Research Institutes, Miaoli County, Taiwan; ${ }^{2}$ Graduate Institute of Pathology, College of Medicine, National Taiwan University, Taipei, Taiwan; ${ }^{3}$ Faculty of Medicine, National Yang Ming University, Taipei, Taiwan; ${ }^{4}$ Department of Psychiatry, Taichung Veterans General Hospital, Taichung, Taiwan; ${ }^{5}$ Institute of Food Science and Technology, National Taiwan University, Taipei, Taiwan; ${ }^{6}$ Department of Life Sciences and Institute of Genome Sciences, National Yang Ming University, Taipei, Taiwan; ${ }^{7}$ Institute of Public Health and Department of Public Health, National Yang Ming University, Taipei, Taiwan; ${ }^{8}$ Department of Industrial Engineering and Engineering Management, College of Engineering, National Tsing-Hua University, HsinChu, Taiwan; ${ }^{9}$ Department of Psychiatry, Yu-Li Veterans Hospital, Hualian County, Taiwan and ${ }^{10} \mathrm{Jianan}$ Mental Hospital, Tainan County, Taiwan 